\title{
Pränatal-Diagnostik und ihre Folgen für Hebammen in der Geburtsvorbereitung

\begin{tabular}{|l|l|l|}
\hline U. & Ulrike & Heil \\
\hline
\end{tabular}

Familienhebamme Gesundheitsamt Wien (MA 15/Reg. 15)

Ulrike Heil, Familienhebamme Gesundheitsamt Wien (MA 15/Ref. 15), Walzengasse 20, A-2380 Perchtoldsdorf

Zum zentralen Thema „Pränataldiagnostik” möchte ich Ihnen einen Blickwinkel präsentieren, der mögli-cherweise einige Überraschungen für Sie bietet.

Um mich zunächst kurz vorzustellen: Mein Name ist Ulrike Heil und ich arbeite als

Familienhebamme des Gesundheitsamtes der Gemeinde Wien in der Geburtsvorbereitung, und in der Betreuung von Risiko-schwangeren als extramurale Betreuungsergänzung.

Bei den Gesprächen mit Schwangeren, egal ob mit oder ohne besonderes Risiko ist es für mich immer wie-der eine traurige Tatsache, daß durch die vielen mögli-chen diagnostischen Maßnahmen viele Ängste nicht nur nicht beseitigt, sondern vielmehr erst ausgelöst werden.

Dabei ist es wohlgemerkt nicht die Untersuchung an sich, die beängstigend ist, sondern die nicht patientengerechte Aufklärung, sei es aus vermeintlichem oder tatsächlichem Zeitmangel, Arbeitsüberlastung Desinteresse oder ganz einfach die Routine.

Es scheint manchmal, als könnten einfache Dinge nicht ohne Fachausdrücke erklärt werden, und die Kon-sequenz davon ist, daß die Frauen zwar belehrt, aber nicht informiert werden.

Bitte halten sie sich vor Augen, daß Begriffe, die für einen damit beruflich Befaßten völlig geläufig sind, für die Frauen Fachchinesisch sind. Vor allem bei pa-thologischen Befunden scheinen die Frauen oft zunächst sehr gefaßt, erst Stunden später beginnen die Schwangeren darüber zu grübeln und als Auskunfts-Quelle fun-giert dann oft eine Freundin, das Elternheft oder etwas ähnliches mit kuriosem Ergebnis.

Besonders der Ultraschall ist ein sehr heißes Thema -oder hätten sie gedacht, daß ein positiver Befund wie die Aussage „großes Kind” ein Problem ist ... Für eine Frau in der 30. Woche, die daraus schließt, daß ihr Kind bereits Geburtsgewicht hat und die meint, bis zur Geburt wird ihr Kind so riesig, daß es vielleicht nicht normal geboren werden kann, ist dieser Befund beängstigend. Wenn sich statt dessen die Formel „ein gut entwickeltes Kind” ein-bürgern würde, könnte auch eine sehr einfache Frau etwas damit anfangen. Laien haben keine Bezugsgròßen, daher ist der Begriff „kleines Kind” oder gar das Wort „Retardiert” auch so besonders problematisch. Wenn noch eine Diskrepanz zwischen Kopf- und Thorax-durchmesser von einer Woche z. B. vorliegt, so ist oft schon massive Angst vor einer vermeintlichen Miß-bildung die Folge und es wird aus Angst vor Bestätigung dieser Vermutung nicht weiter nachgefragt. Leider kommt noch sehr oft eine unbedachte Äußerung des Untersuchers dazu, und für die Frau ist das Unglück fertig, und auch nachfolgende Aufklärung kann nur noch abschwächen, aber den Verdacht nicht völlig entkräften. Ein Beispiel aus der Praxis dazu zur Illustration: Eine 25jährige Erstgebärende wird wegen fraglicher Placentainsuffizienzen in der 36. SSW an eine Klinik zur Doppleruntersuchung zugewiesen. Bei dieser Untersuchung spricht der Arzt den Verdacht einer Trisomie 16 und 18 aus. Die Frau hat daraufhin die Klinik gewechselt, dort wurde kein 
verdächtiger Befund erhoben, aber die Diagnose „kleines Kind” bestätigt. Wie ich bei einem Hausbesuch feststellte, war die Frau strenge Vegetarierin, nur nach den Lebensgewohnheiten hatte vorher niemand gefragt, und ihre Sorgen in den letzten 4 Wochen können sie sich auch mit wenig Phantasie vorstellen. Übrigens hat sie ein gesundes Kind mit 2800 g geboren. Fast bei jeder Geburtsvorbereitung, zumindest aber bei jeder Gruppe mehrmals werden Ultraschallbefunde gezeigt mit der Bitte um Erklärung. Ähnlich verhält es sich mit der Dopplersonographie, allerdings wird dabei vorallem als beunruhigend empfunden, daß die Befun-dung mitunter lang dauern kann. Dann wird der Verdacht gehegt, es sei „etwas nicht in Ordnung”, und man will es der werdenden Mutter nur nicht sagen. Das CTG ist das nächste Reizwort, wobei eigentlich in erster Linie der hohe Zeitaufwand und die langen War-tezeiten in den Ambulanzen vor allem für Mehr-gebärende zum Problem wird. Mir haben mehrmals Frauen gesagt: „Wenn man bei meinem ersten Kind auch so ein Theater gemacht hätte, so hätt’s kein zweites gege-ben!” Ein wörtliches Zitat, das zu denken gibt! Wirklich besorgt sind die Frauen eigentlich nur vor einer frühen Aufnahme ins Krankenhaus, wenn sie sich selbst wohlfühlen.

Ich habe immer wieder die Beobachtung gemacht, daß hingegen CTG'Kontrollen zuhause, verbunden mit einem intensiven Gespräch über die ganz persönliche Situation der Frau nicht nur sehr positiv aufgenommen wurde, sondern auch für die Frauen deutlich beruhigend sind und dadurch zu einer positiven Beeinflussung der ganzen Lage führten, die Frauen fühlen sich sicherer und als Mensch und zwar als ganzer angenommen.

Obwohl zu diesem Thema noch soviel zu sagen ware, möchte ich ihnen nur ein paar Dinge ans Herz legen:

Sprechen Sie mit den Frauen, reden Sie deutsch, Sie haben Laien vor sich und die Unwissenheit der Frauen ist auch im Zeitalter der Sexualkunde oft derart, daß einem der Mund offen steht. Umso wichtiger ist, daß auch scheinbare Selbstverständlichkeiten erwähnt werden.

Wählen Sie Ihre Worte vorsichtig und unmißver-ständlich und haben Sie Geduld, sonst verlieren die Frauen das Vertrauen, und das macht sprachlos und, wenn dann Maßnahmen mit entsprechender Bedacht-samkeit eingesetzt werden, wird auch das Ergebnis der Schwangerenbetreuung zufriedenstellend sein.

Nicht die Quantität entscheidet, sondern die Qualität.

Für uns Hebammen hat die Praenataldiagnostik den Vorteil, die Frauen gezielt beraten zu können; gleichzeitig ist der Zeitaufwand für Aufklärung und Angstbekämp-fung nicht zu unterschätzen und die laiengerechte Befund-aufklärung schon ein fester Bestandteil des Programmes.

104

Heil

Pränatal-Diagnostik und ihre Folgen für Hebammen in der Geburtsvorbereitung 